\title{
Gaia DR2 view of the Lupus V-VI clouds: The candidate diskless young stellar objects are mainly background contaminants
}

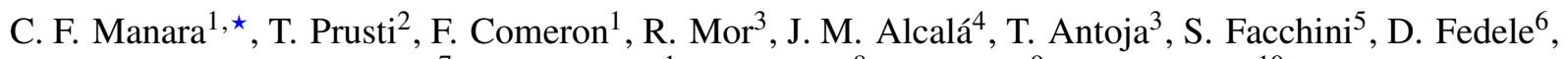 \\ A. Frasca ${ }^{7}$, T. Jerabkova ${ }^{1}$, G. Rosotti ${ }^{8}$, L. Spezzi ${ }^{9}$, and L. Spina ${ }^{10}$ \\ ${ }^{1}$ European Southern Observatory, Karl-Schwarzschild-Strasse 2, 85748 Garching bei München, Germany \\ e-mail: cmanara@eso.org \\ 2 Scientific Support Office, Directorate of Science, European Space Research and Technology Centre (ESA/ESTEC), Keplerlaan 1, \\ 2201 AZ Noordwijk, The Netherlands \\ 3 Institut de Ciències del Cosmos, Universitat de Barcelona (IEEC-UB), Martí i Franquès 1, 08028 Barcelona, Spain \\ 4 INAF-Osservatorio Astronomico di Capodimonte, via Moiariello 16, 80131 Napoli, Italy \\ 5 Max-Planck-Institut für Extraterrestrische Physik, Giessenbachstrasse 1, 85748 Garching bei München, Germany \\ 6 INAF-Osservatorio Astrofisico di Arcetri, L.go E. Fermi 5, 50125 Firenze, Italy \\ 7 INAF-Osservatorio Astrofisico di Catania, via S. Sofia, 78, 95123 Catania, Italy \\ 8 Institute of Astronomy, University of Cambridge, Madingley Road, Cambridge CB3 0HA, UK \\ 9 European Organisation for the Exploitation of Meteorological Satellites (Eumetsat), Eumetsat Allee 1, 64295 Darmstadt, Germany \\ 10 Monash Centre for Astrophysics, School of Physics and Astronomy, Monash University, VIC 3800, Australia
}

Received 7 May 2018 / Accepted 11 June 2018

\begin{abstract}
Extensive surveys of star-forming regions with Spitzer have revealed populations of disk-bearing young stellar objects. These have provided crucial constraints, such as the timescale of dispersal of protoplanetary disks, obtained by carefully combining infrared data with spectroscopic or X-ray data. While observations in various regions agree with the general trend of decreasing disk fraction with age, the Lupus V and VI regions appeared to have been at odds, having an extremely low disk fraction. Here we show, using the recent Gaia data release 2 (DR2), that these extremely low disk fractions are actually due to a very high contamination by background giants. Out of the 83 candidate young stellar objects (YSOs) in these clouds observed by Gaia, only five have distances of $\sim 150 \mathrm{pc}$, similar to YSOs in the other Lupus clouds, and have similar proper motions to other members in this star-forming complex. Of these five targets, four have optically thick (Class II) disks. On the one hand, this result resolves the conundrum of the puzzling low disk fraction in these clouds, while, on the other hand, it further clarifies the need to confirm the Spitzer selected diskless population with other tracers, especially in regions at low galactic latitude like Lupus V and VI. The use of Gaia astrometry is now an independent and reliable way to further assess the membership of candidate YSOs in these, and potentially other, star-forming regions.
\end{abstract}

Key words. stars: pre-main sequence - stars: formation - astrometry

\section{Introduction}

The timescale on which protoplanetary disks evolve is a key constraint on planet formation and disk evolution models. Observations show that the fraction of young stellar objects (YSOs) surrounded by optically thick disks decreases exponentially with time with a typical timescale of $\sim 2-3 \mathrm{Myr}$ (e.g., Haisch et al. 2001; Hernández et al. 2007; Fedele et al. 2010).

In this context, the very low disk fraction of about $15 \%$ derived with Spitzer in the Lupus V and VI clouds by Spezzi et al. (2011), significantly smaller than the value of about 50\% (Merín et al. 2008) for the nearby 2-3-Myr-old (Alcalá et al. 2014; Frasca et al. 2017) Lupus I and III clouds, appears to be at odds with the general trend. Indeed, if the age of these clouds is compatible with that of the other parts of the Lupus complex, and the candidate diskless (Class III) YSOs are confirmed to be members of the cloud population, other processes would be required to explain the particularly fast dispersal of disks

\footnotetext{
$\star$ ESO Fellow.
}

(Spezzi et al. 2011), such as external photoevaporation or higher binarity fraction (e.g., Facchini et al. 2016; Rosotti \& Clarke 2018). Since the Class III population of Spitzer-selected candidate YSOs is known to be contaminated by background objects with contaminant fractions between 25 and 90\% (Dunham et al. 2015), independent data must be used to confirm the YSO status of the candidates. An analysis of 31 of these YSO candidates in these two clouds, conducted by Romero et al. (2012) using optical spectroscopy, showed that this sub-sample of the population is made up of background contaminants. In a re-analysis of the same Spitzer data by Dunham et al. (2015), 11 objects in Lupus V and 17 in Lupus VI were classified as likely asymptotic giant branch (AGB) contaminants. However, the majority of the Spitzer candidates are still to date not confirmed as legitimate YSOs.

Here we adopt another powerful method, independent of the one used by Romero et al. (2012), to discriminate members of the star-forming region from background contaminants. We explore the astrometric parameters for the whole YSO candidate population of the Lupus V and VI clouds from the 
recent Gaia (Gaia Collaboration 2016) data release 2 (DR2, Gaia Collaboration 2018a; Lindegren et al. 2018). Using their parallax, as well as their proper motions and location on the color-magnitude diagram, we can determine the real nature of the candidate YSOs in these clouds to further constrain how protoplanetary disks evolve.

\section{Sample and data}

The Lupus cloud complex is a well-known nearby low-mass star-forming complex located at a distance of $\sim 150-200 \mathrm{pc}$ (Comerón 2008; Gaia Collaboration 2018a). It is composed of several molecular clouds, whose density peaks, or clumps, are usually referred to as distinct clouds. In particular, the largest CO molecular cloud of the complex contains the Lupus III, IV, V, and VI clouds (Tachihara et al. 2001). Lupus was one of the targets of the Spitzer Legacy surveys From Molecular Cores to Planet-forming Disk (c2d, PI: N. Evans; Evans et al. 2009) and Gould's Belt (GB, PI: L. Allen; Dunham et al. 2015). Among the various regions targeted in these surveys, the list of YSO candidates from which Spezzi et al. (2011) derived the comparably low disk fraction includes 43 and 45 objects in the Lupus V and VI clouds, respectively. The analysis of the same dataset by Dunham et al. (2015) confirmed the candidate YSO status for 32 and 28 of these targets, respectively, while suggesting that the remaining objects are likely AGB contaminants. Furthermore, they suggest that the contaminant fraction among the Class III YSO candidates could be as high as $90 \%$. In this work, we use the complete initial list by Spezzi et al. (2011) for the analysis.

\subsection{Gaia data collection}

We have downloaded the data from the Gaia archive using the ADQL queries reported in Appendix A. We first select all objects within $243.5^{\circ}<\mathrm{RA}<246.9^{\circ}$ and $-38.5^{\circ}<$ Dec $<-36.3^{\circ}$ (Lupus V) and $244.4^{\circ}<\mathrm{RA}<247.8^{\circ}$ and $-42.1^{\circ}<$ Dec $<-38.7^{\circ}$ (Lupus VI). These searches find, in the Gaia DR2 catalog, a total of 663071 targets in the Lupus V region of the sky and 1517387 in the Lupus VI field. We then use the cross-match of the Gaia catalog with the 2MASS catalog provided by the Gaia consortium within the Gaia DR2 to find the best match within radii of $1^{\prime \prime}$ (see Appendix A), and find, in the same regions of the sky, 188053 matches for the Lupus V cloud region, and 440565 for the Lupus VI region.

We then proceed by matching the catalog of candidate YSOs by Spezzi et al. (2011) with the Gaia DR2 catalog. We check that the matches between Gaia and the catalog by Spezzi et al. (2011) are always with angular separations $\lesssim 0.3^{\prime \prime}$. We find a total of 39 out of 43 matches for the Lupus V region, and 44 out of 45 for the Lupus VI region. The objects with no Gaia counterpart are the faintest in the samples $(J \sim 13-16 \mathrm{mag}, K \sim 15 \mathrm{mag})$. The one candidate YSO in Lupus VI with no match is classified by Spezzi et al. (2011) as Flat source, while three of the four targets with no match in Lupus V are classified as Class III, and the last one as Class II based on their Spitzer infrared(IR)-excess up to $24 \mu \mathrm{m}$.

\section{Analysis of Gaia data}

The first indication that an object is a member of the Lupus complex is found by examining its parallax. The distribution of parallaxes for the YSO candidates in these two clouds is shown in Fig. 1. Of these targets, only five objects in Lupus V have
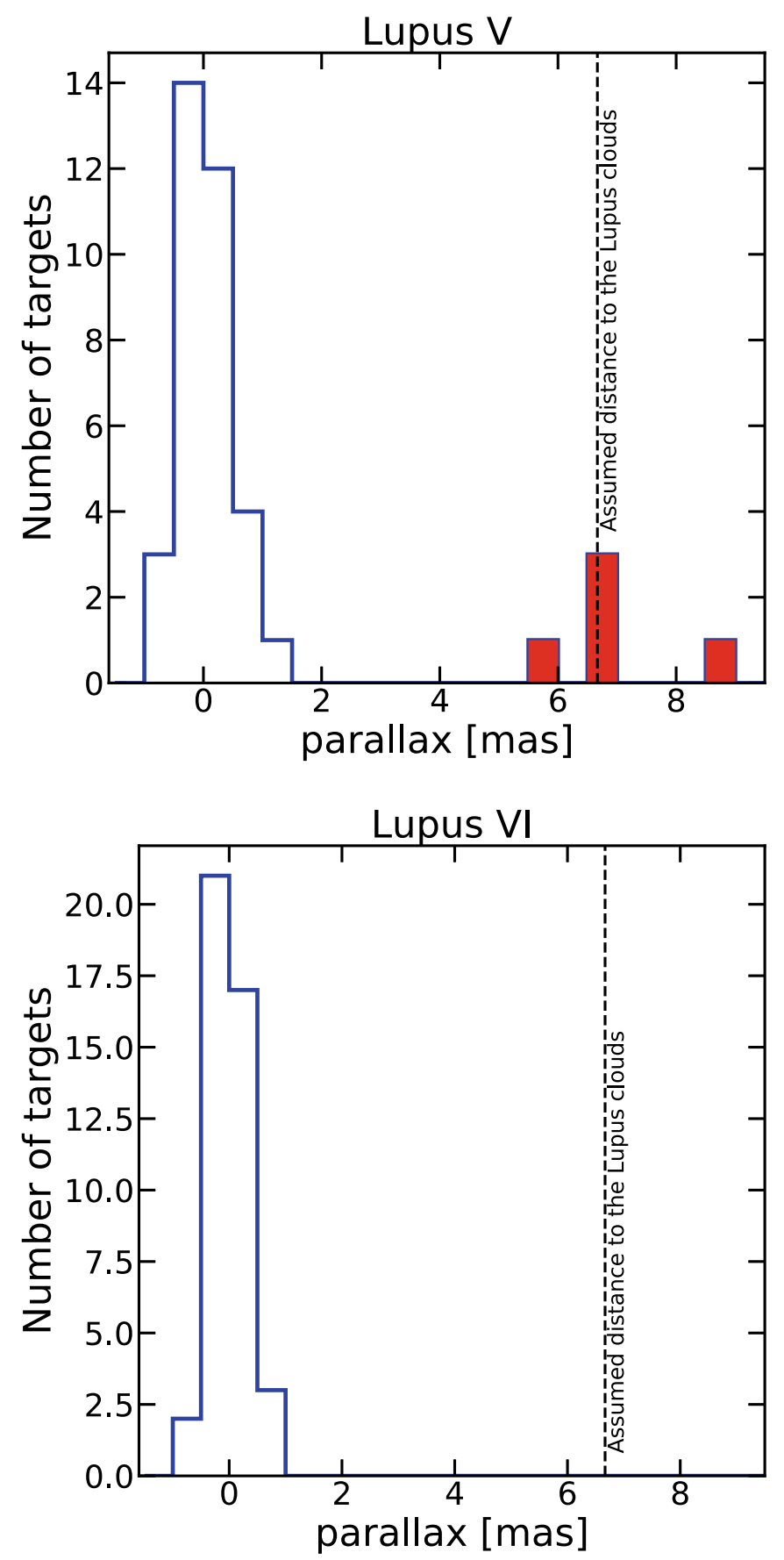

Fig. 1. Histogram of measured parallaxes for the candidate YSOs proposed by Spezzi et al. (2011) in the Lupus V (top) and Lupus VI (bottom) clouds. Highlighted in red are the objects with parallax $>5$ mas. These are five objects in the Lupus V cloud, which are the only real YSOs among the Spitzer-selected candidates.

parallaxes larger than 5 mas, corresponding to distances smaller than $200 \mathrm{pc}$, while all the other objects have parallax $\lesssim 2$ mas, implying distances larger than $500 \mathrm{pc}$. No candidate YSOs in the Lupus VI region have parallax $>1$ mas. This information already suggests that most of the candidate YSOs selected by Spezzi et al. (2011) are, in reality, background objects.

We also explored whether there is any dependence of the measured parallaxes of the YSO candidates with their position in the sky, and we find that the five objects with parallaxes larger than 5 mas are all in low-extinction parts of the cloud, 


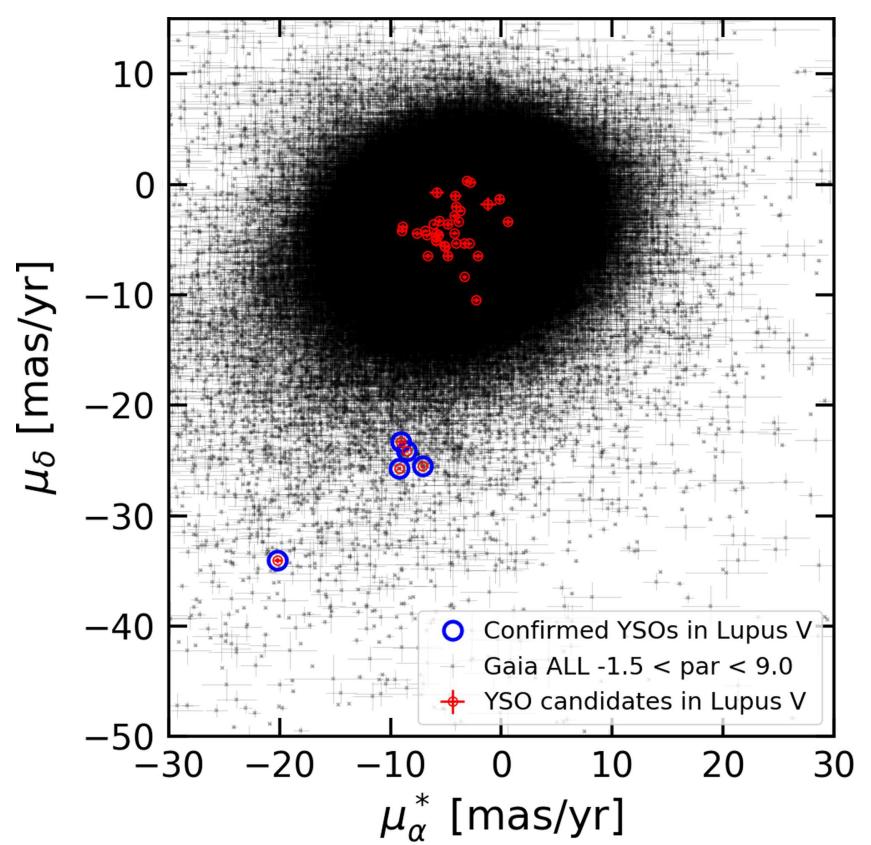

Fig. 2. Proper motion of the Gaia targets in the field of view of the Lupus $\mathrm{V}$ region, highlighting the candidate young stellar objects with red circles, and the five confirmed young stars with cyan circles.

mainly on the west side, in the direction of the Lupus III cloud (see Appendix B). We also find that four of the YSO candidates with parallaxes larger than 5 mas, and therefore distances smaller than $200 \mathrm{pc}$, are classified as Class II by Spezzi et al. (2011), and that the remaining one is classified as Class III. This classification is confirmed also by Dunham et al. (2015), and none of these five targets are flagged as likely being AGB contaminants. These five objects have a weighted mean parallax of $6.662 \pm 0.013$ mas, corresponding to a mean distance of $\sim 150$ pc. If the Lupus clouds are at 150 pc (e.g., Comerón 2008), then three of these targets are in the cloud, while one is in front of it and one behind it.

To further investigate whether the closer YSO candidates are different from the background objects, we show in Fig. 2 the proper motion for the YSO candidates in the Lupus V cloud. Four out of the five objects with parallaxes larger than 5 mas are all clustered at $\mu_{\alpha}^{*} \sim-9$ mas $\mathrm{yr}^{-1}, \mu_{\delta} \sim-25 \mathrm{mas} \mathrm{yr}^{-1}$, and the last

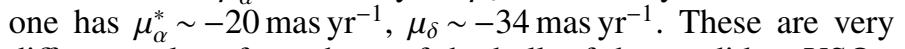
different values from those of the bulk of the candidate YSOs,

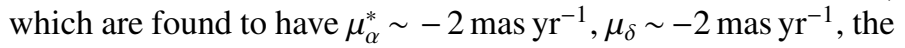
same parameter space as most of the Gaia targets in the field of view, which are mainly background objects. The difference in the proper motion space of these closer YSO candidates suggests that they are a homogeneous group that is kinematically distinct from the more distant objects. This confirms that they most likely belong to the cloud population. When comparing the proper motion values for the five close-by targets with those of other confirmed YSOs in the Lupus complex (López Martí et al. 2011), these are found in very good agreement with those with higher proper motion in this region. This confirms these five objects as members of the Lupus star-forming region.

Finally, we collect the $G, B_{P}, R_{P}$ magnitudes for all the targets included in the Gaia DR2 catalog. Following Gaia Collaboration (2018b), we convert the $G$ magnitudes into absolute magnitudes as $M_{G}=G+5+5 \log _{10}(\omega / 1000)$, with $\omega$ being the parallax in milliarcseconds. Since this works only for targets with measured magnitudes and positive parallaxes, the
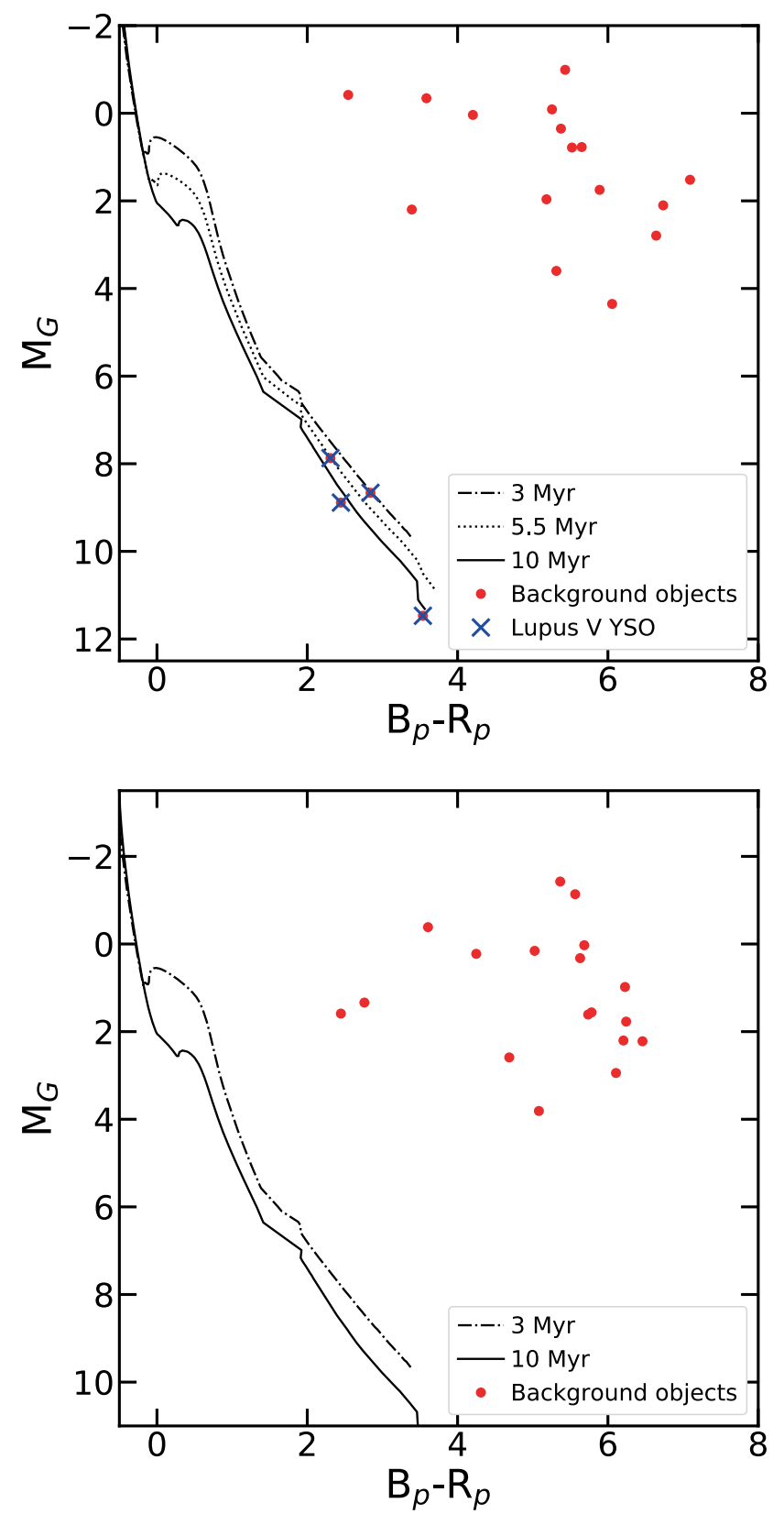

Fig. 3. Absolute magnitude vs color for the candidate YSOs in the Lupus V (top) and Lupus VI (bottom) regions with positive parallax and available Gaia magnitudes. Only the four confirmed young objects in Lupus V with available Gaia magnitudes have color and magnitudes compatible with age $<10 \mathrm{Myr}$, according to the overplotted extinctionfree isochrones.

absolute magnitude versus color plots in Fig. 3 show only a fraction of the YSO candidates. Nevertheless, the comparison of the position of the YSO candidates on these color-magnitude diagrams, especially when compared with the isochrones by Marigo et al. (2017), shows that four out of five of the nearby targets in Lupus $\mathrm{V}$ are indeed young objects with isochronal ages between 3 and $10 \mathrm{Myr}$, while all the other YSO candidates are instead located in the part of the color-magnitude diagram compatible with the locus of giants. No $B_{P}$ and $R_{P}$ magnitudes are available for one of the five bona fide YSOs in Lupus V. Neglecting the effect of interstellar extinction has a minor impact, as these young objects are all located in regions of the clouds where $E(B-V)$ is smaller than 1.4 mag (see Appendix B), 
Table 1. Properties of the confirmed YSOs in the Lupus V region.

\begin{tabular}{|c|c|c|c|c|c|c|c|}
\hline 2MASS J & \multicolumn{2}{|c|}{ Gaia DR2 ID } & \multicolumn{2}{|c|}{$\begin{array}{l}\text { RA } \\
\text { (deg) }\end{array}$} & \multicolumn{2}{|l|}{$\begin{array}{l}\text { Dec } \\
\text { (deg) }\end{array}$} & $\begin{array}{c}\text { parallax } \\
\text { (mas) }\end{array}$ \\
\hline $16191403-3747280$ & \multicolumn{2}{|c|}{6021420630046381440} & \multicolumn{2}{|c|}{244.80843901} & \multicolumn{2}{|c|}{-37.79119223} & $6.568 \pm 0.042$ \\
\hline $16172485-3657405$ & \multicolumn{2}{|c|}{6021662385163162240} & \multicolumn{2}{|c|}{244.35352824} & \multicolumn{2}{|c|}{-36.96138759} & $6.760 \pm 0.067$ \\
\hline \multirow{2}{*}{$16172475-3657332$} & \multicolumn{2}{|c|}{6021662385163163648} & \multicolumn{2}{|c|}{244.35306973} & \multicolumn{2}{|c|}{-36.95935461} & $6.588 \pm 0.082$ \\
\hline & \multicolumn{2}{|c|}{6021805356032645504} & \multicolumn{2}{|c|}{244.32543416} & \multicolumn{2}{|c|}{-36.77529065} & $5.957 \pm 0.179$ \\
\hline \multirow{7}{*}{ 16192684-3651235 } & \multicolumn{2}{|c|}{6021745462701109376} & \multicolumn{2}{|c|}{244.86172975} & \multicolumn{2}{|c|}{-36.85671498} & $8.910 \pm 0.187$ \\
\hline & $\begin{array}{c}\mu_{\alpha}^{*} \\
\left(\mathrm{mas} \mathrm{yr}^{-1}\right)\end{array}$ & $\begin{array}{c}\mu_{\delta} \\
\left(\mathrm{mas} \mathrm{yr}^{-1}\right)\end{array}$ & $\begin{array}{c}G \\
(\mathrm{mag})\end{array}$ & $\begin{array}{c}B_{P} \\
(\mathrm{mag})\end{array}$ & $\begin{array}{cc} & R_{P} \\
(\mathrm{mag})\end{array}$ & $\begin{array}{l}\text { Lada } \\
\text { Class }\end{array}$ & \\
\hline & $-8.56 \pm 0.10$ & $-24.10 \pm 0.06$ & 13.78 & 14.93 & 12.62 & II & \\
\hline & $-9.21 \pm 0.14$ & $-25.68 \pm 0.08$ & 14.51 & 16.12 & 13.28 & III & \\
\hline & $-7.14 \pm 0.18$ & $-25.50 \pm 0.10$ & 14.79 & 16.06 & 13.61 & II & \\
\hline & $-9.03 \pm 0.36$ & $-23.32 \pm 0.20$ & 17.64 & .. & .. & II & \\
\hline & $-20.18 \pm 0.33$ & $-34.04 \pm 0.24$ & 16.72 & 18.87 & 15.33 & II & \\
\hline
\end{tabular}

Notes. Reference epoch: 2015.5. Lada classes are from Spezzi et al. (2011).

and this would just change the isochronal age of the objects by $\lesssim 1$ Myr (Table 1).

\section{Discussion}

Only five out of 83 candidate YSOs in the Lupus V and VI clouds are confirmed YSOs. These five targets have similar proper motions to other YSOs in the Lupus complex and four of them have IR excess typical of Class II objects. They could either be a different population with respect to the nearby Lupus III cloud, or members of the latter. If they are an independent population, then the disk fraction found here could seem too high for their age (some millions of years). However, the age estimate is subject to large uncertainties (e.g., Soderblom et al. 2014) and we cannot asses whether we have a complete census of this putative YSO population of Lupus V. It is to be expected that a larger population of diskless YSOs is also present in this cloud. The location of the clouds being to the east supports instead the hypothesis that these objects are members of Lupus III, and they are located off-cloud, like the YSOs studied by Comerón et al. (2009).

The lack of confirmed YSOs in Lupus VI and the small number of confirmed YSOs in Lupus V could imply that either star formation has not started in these clouds, like in the Musca cloud north of the Chamaeleon complex (Gregorio Hetem et al. 1988; Cox et al. 2016), or that these clouds are actually relatively sterile. Spezzi et al. (2011) discussed the fact that the extinction maps for these regions peak at $A_{V} \lesssim 6 \mathrm{mag}$, which is in the range of values where star formation is highly inefficient (Lada et al. 2013). Therefore, it is indeed possible that these clouds are not able to form stars now.

This work advocates caution when dealing with the conclusions derived from disk statistics based on large numbers of Spitzer-selected Class III objects, which are prone to contamination by unrelated types of objects, as extensively discussed also by Dunham et al. (2015). This effect is lower in regions dominated by a high density of Class II YSOs, which are selected with a much higher level of confidence by Spitzer color criteria. Indeed, the spectroscopic follow-up of Spitzer surveys carried out by, for example, Oliveira et al. (2009) in the Serpens region, or Alcalá et al. $(2014,2017)$ in the Lupus I, III, and IV clouds, looking for the presence of lithium, $\mathrm{H} \alpha$, and several other indicators of youth, have shown that about $95 \%$ of the
Spitzer Class II candidates are confirmed as legitimate YSOs. Only a handful of contaminant background giants are found in these regions (see also Dunham et al. 2015; Frasca et al. 2017). In the case of Lupus V and VI, however, the candidate YSOs were mainly Class III, and also Oliveira et al. (2009), as well as Romero et al. (2012) and Dunham et al. (2015), have shown that the fraction of contaminants can be larger than $50 \%$ for diskless stars. Furthermore, the low location close to the galactic plane of the Lupus $\mathrm{V}$ cloud $\left(b \sim 8.8^{\circ}\right)$, and the even lower location of the Lupus VI cloud $\left(b \sim 6.3^{\circ}\right)$, implies that contamination from background giants can be much higher than in star-forming regions well away from the galactic plane, as also pointed out by Dunham et al. (2015). We verified the number of background giants expected in the Gaia catalog in these regions of the sky by exploring the content of the Gaia Universe Model Snapshot (GUMS; Robin et al. 2012). We selected all the stars with $G<21.5 \mathrm{mag}$ from the same region of the sky as the one that we queried from the Gaia archive (see Sect. 2). The number of selected stars is of the same order of magnitude as those found in the Gaia archive. By exploring the color-magnitude diagram for these targets, we found that there are $\sim 3 \times 10^{4}$ and $\sim 10^{5}$ background giants in these fields, of which the majority $(\sim 65-75 \%)$ are red giants. Furthermore, the number of background contaminants found here is of the order of the estimated red giants of spectral types M7, M8, M9, C-type and S-type in the Lupus V and Lupus VI fields predicted by GUMS, that is, 50 and 150 in the Lupus V and Lupus VI fields, respectively. These are the reddest giants, and are the likely contaminants of the Spitzer YSO selection criteria. This means that the number of background giants erroneously classified as Class III YSO candidates in previous Spitzer surveys of these regions is just a tiny fraction of the total number of background objects. This implies, on the one hand, that the high fraction of contaminants present in these samples is in line with the number of the reddest giants, while there are orders of magnitude more giants in the field. On the other hand, this also implies that not all of the background objects are classified with Spitzer as Class III YSO candidates, as many are correctly excluded by the color-color selection criteria.

On top of spectroscopy and the detection of X-ray emission from these YSO candidates, we have shown here that the analysis of the astrometric properties of the targets obtained with Gaia is a powerful tool to discriminate between bona fide YSOs and contaminants. 


\section{Conclusions}

Here, we investigate the astrometric properties of the Spitzer selected candidate YSOs in the Lupus V and VI clouds and find that only five targets have parallaxes larger than 5 mas, proper motions compatible with other YSOs in the Lupus complex, and occupy the right region of the color-magnitude diagram to be considered YSOs. Of these five objects, four are shown to have optically thick disks based on their Spitzer colors. This reconciles the disk fraction in Lupus V-VI with the other regions in the Lupus clouds complex, without the need to invoke an exceptionally short disk lifetime. These targets are located at $d \sim 150 \mathrm{pc}$ and have ages between 3 and $10 \mathrm{Myr}$. These objects are either members of the Lupus V region, or off-cloud YSOs of Lupus III. In the former case, it is possible that several Class III objects in this region have not yet been detected by previous surveys. Furthermore, these two clouds, to our knowledge only hosting a total of five YSOs, are possibly sterile or have not yet begun forming stars.

The remaining 78 YSO candidates in these clouds present in the Gaia DR2 catalog are not YSOs, but background objects, mainly red giants. Previous claims of an anomalously low disk fraction can therefore be explained by the very low star formation activity in Lupus V and VI, the low reliability of selection criteria based on Spitzer colors when applied to Class III sources, and by the low galactic latitude of both clouds, which increases the areal density of potential contaminants.

The work presented here highlights the power of the astrometric information supplied by Gaia as a new fundamental tool to obtain reliable samples of bona fide members of nearby starforming regions.

Acknowledgements. We thank the anonymous referee for the constructive report which helped to improve the quality of the work. This work has made use of data from the European Space Agency (ESA) mission Gaia (https://www . cosmos.esa.int/gaia), processed by the Gaia Data Processing and Analysis Consortium (DPAC, https://www. cosmos.esa.int/web/gaia/dpac/ consortium). Funding for the DPAC has been provided by national institutions, in particular the institutions participating in the Gaia Multilateral Agreement We made use of the Python packages numpy, astropy, aplpy, and matplotlib for the analysis. CFM acknowledges support with an ESO Fellowship. JMA acknowledge financial support from the project PRIN-INAF 2016 The Cradle of Life-GENESIS-SKA (General Conditions in Early Planetary Systems for the rise of life with SKA). D.F. and acknowledge support from the Italian Ministry of Education, Universities and Research, project SIR (RBSI14ZRH). This work has been supported by the DISCSIM project, grant agreement 341137 funded by the European Research Council under ERC-2013-ADG.

\section{References}

Alcalá, J. M., Natta, A., Manara, C. F., et al. 2014, A\&A, 561, A2 Alcalá, J. M., Manara, C. F., Natta, A., et al. 2017, A\&A, 600, A20 Comerón, F. 2008, Handbook of Star Forming Regions, Vol. II, 5, 295 Comerón, F., Spezzi, L., \& López Martí, B. 2009, A\&A, 500, 1045 Cox, N. L. J., Arzoumanian, D., André, P., et al. 2016, A\&A, 590, A110 Dunham, M. M., Allen, L. E., Evans, N. J., II, et al. 2015, ApJS, 220, 11 Evans, N. J., II, Dunham, M. M., Jørgensen, J. K., et al. 2009, ApJS, 181, 321 Facchini, S., Clarke, C. J., \& Bisbas, T. G. 2016, MNRAS, 457, 3593 Fedele, D., van den Ancker, M. E., Henning, T., Jayawardhana, R., \& Oliveira, J. M. 2010, A\&A, 510, A72

Frasca, A., Biazzo, K., Alcalá, J. M., et al. 2017, A\&A, 602, A33

Gaia Collaboration (Prusti, T., et al.) 2016, A\&A, 595, A1

Gaia Collaboration (Brown, A. G. A., et al.) 2018a, A\&A, in press, DOI: 10.1051/0004-6361/201833051

Gaia Collaboration (Babusiaux, C., et al.) 2018b, A\&A, in press, DOI: $10.1051 / 0004-6361 / 201832843$

Gregorio Hetem, J. C., Sanzovo, G. C., \& Lepine, J. R. D. 1988, A\&AS, 76, 347

Haisch, K. E. Jr., Lada, E. A., \& Lada, C. J. 2001, ApJ, 553, L153

Hernández, J., Hartmann, L., Megeath, T., et al. 2007, ApJ, 662, 1067

Lada, C. J., Lombardi, M., Roman-Zuniga, C., et al. 2013, ApJ, 778, 133

Lindegren, L., Hernandez, J., Bombrun, A., et al. 2018, A\&A, in press, DOI: $10.1051 / 0004-6361 / 201832727$

López Martí, B., Jiménez-Esteban, F., \& Solano, E. 2011, A\&A, 529, A108

Luri, X., Brown, A. G. A., Sarro, L. M., et al. 2018, A\&A, in press, DOI: $10.1051 / 0004-6361 / 201832964$

Marigo, P., Girardi, L., Bressan, A., et al. 2017, ApJ, 835, 77

Merín, B., Jørgensen, J., Spezzi, L., et al. 2008, ApJS, 177, 551

Oliveira, I., Merín, B., Pontoppidan, K. M., et al. 2009, ApJ, 691, 672

Prato, L., Rice, E. L., \& Dame, T. M. 2008, Handbook of Star Forming Regions, Vol. I, 4, 18

Robin, A. C., Luri, X., Reylé, C., et al. 2012, A\&A, 543, A100

Romero, G. A., Schreiber, M. R., Cieza, L. A., et al. 2012, ApJ, 749, 79

Rosotti, G. P., \& Clarke, C. J. 2018, MNRAS, 473, 5630

Schlegel, D. J., Finkbeiner, D. P., \& Davis, M. 1998, ApJ, 500, 525

Soderblom, D. R., Hillenbrand, L. A., Jeffries, R. D., Mamajek, E. E., \& Naylor, T. 2014, Protostars and Planets VI, 219

Spezzi, L., Vernazza, P., Merín, B., et al. 2011, ApJ, 730, 65

Tachihara, K., Toyoda, S., Onishi, T., et al. 2001, PASJ, 53, 1081 


\section{Appendix A: ADQL queries}

Part of sky of Lupus V:

SELECT

gaia.solution_id, gaia.designation, gaia.source_id, gaia.ref_epoch, gaia.ra, gaia.ra_error, gaia.dec, gaia.dec_error, gaia.parallax, gaia.parallax_error, gaia.parallax_over_error, gaia.pmra, gaia.pmra_error, gaia.pmdec, gaia.pmdec_error, gaia.astrometric_gof_al, gaia.astrometric_params_solved, gaia.phot_g_mean_mag, gaia.phot_bp_mean_mag, gaia.phot_rp_mean_mag, gaia.radial_velocity, gaia.radial_velocity_error FROM gaiadr2.gaia_source as gaia

WHERE

gaia.dec $>-38.5$ AND gaia.dec $<-36.3$

AND gaia.ra $>243.5$ AND gaia.ra $<246.9$

Part of sky of Lupus VI:

SELECT

gaia.solution_id, gaia.designation, gaia.source_id, gaia.ref_epoch, gaia.ra, gaia.ra_error, gaia.dec, gaia.dec_error, gaia.parallax, gaia.parallax_error, gaia.parallax_over_error, gaia.pmra, gaia.pmra_error, gaia.pmdec, gaia.pmdec_error, gaia.astrometric_gof_al, gaia.astrometric_params_solved, gaia.phot_g_mean_mag, gaia.phot_bp_mean_mag, gaia.phot_rp_mean_mag, gaia.radial_velocity, gaia.radial_velocity_error

FROM gaiadr2.gaia_source as gaia

WHERE

gaia.dec $>-42.1$ AND gaia.dec $<-38.7$

AND gaia.ra $>244.4$ AND gaia.ra $<247.8$

And also the following data cross-matched with 2MASS:

\section{SELECT}

FROM gaiadr2.gaia_source as gaia

INNER JOIN gaiadr2.tmass_best_neighbour as xmatch ON gaia.source_id = xmatch.source_id

INNER JOIN gaiadr1.tmass_original_valid as tmas ON tmas.tmass_oid = xmatch.tmass_oid

WHERE

gaia.dec $>-38.5$ AND gaia.dec $<-36.3$

AND gaia.ra $>243.5$ AND gaia.ra $<246.9$

AND xmatch.angular_distance $<1.0$

SELECT

FROM gaiadr2.gaia_source as gaia

INNER JOIN gaiadr2.tmass_best_neighbour as xmatch ON gaia.source_id = xmatch.source_id

INNER JOIN gaiadr1.tmass_original_valid as tmas ON tmas.tmass_oid = xmatch.tmass_oid

WHERE

gaia.dec $>-42.1$ AND gaia.dec $<-38.7$

AND gaia.ra $>244.4$ AND gaia.ra $<247.8$

AND xmatch.angular_distance $<1.0$

\section{Appendix B: Parallaxes as a function of position in the sky}

Here we explore whether there is any dependence of the measured parallax on the position of the objects in the sky, which could imply that only portions of the clouds contain YSOs. Figure B.1 shows that there is no trend of parallaxes with declination, and that the five targets with parallax $>5$ are, in fact, located at different declinations within the Lupus V cloud. Instead, Fig. B.2 shows that all the objects with parallax $>5$ mas are on the west side of the cloud. In these figures, the YSO candidates classified as Class II objects are highlighted with a blue cross.

We show the location of the candidate YSOs and the actual members in Fig. B.3 superimposed on the extinction map by Schlegel et al. $(1998)^{1}$. The members are found to be in the lower extinction part of the cloud, and mainly on the west side of it, thus in the direction of the Lupus III cloud.

1 Obtained at https://irsa.ipac.caltech.edu/applications/DUST/ 

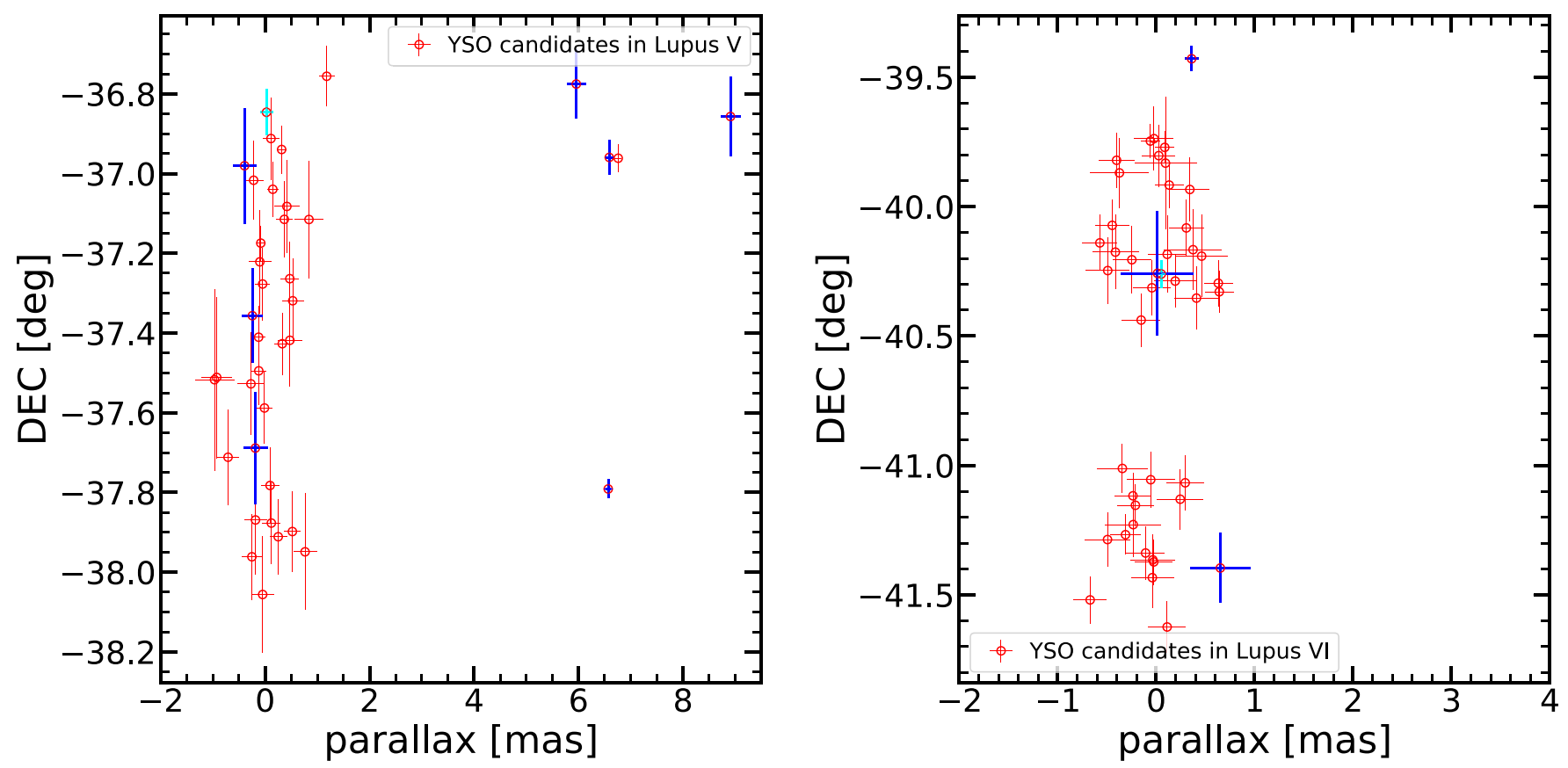

Fig. B.1. Parallaxes of candidate YSOs as a function of declination for objects in the Lupus V (left) and Lupus VI (right) region of the sky. Blue crosses indicate candidate Class II objects, cyan crosses candidate transition disk objects.
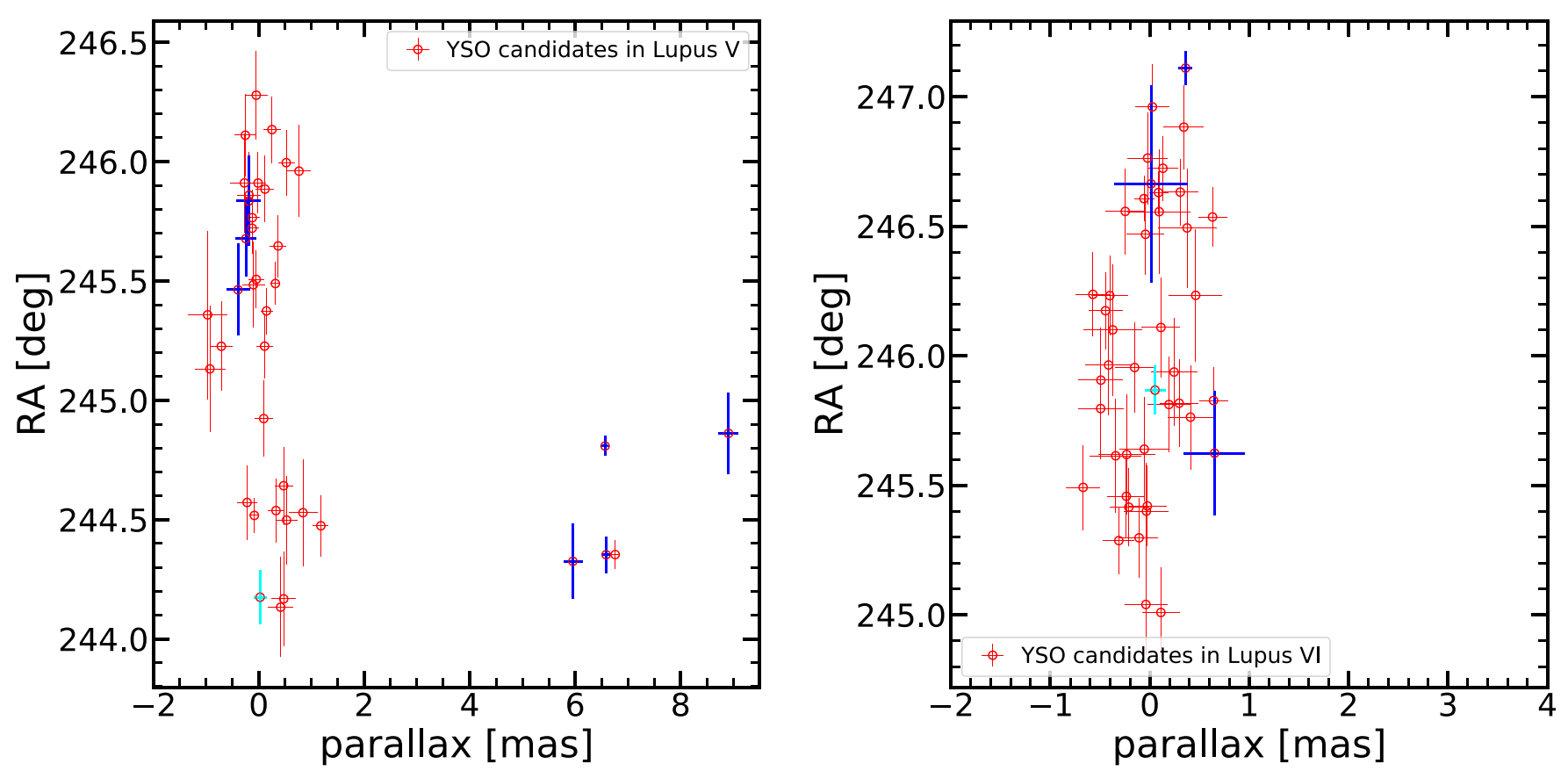

Fig. B.2. Parallaxes of candidate YSOs as a function of right ascension for objects in the Lupus V (left) and Lupus VI (right) region of the sky. Blue crosses indicate candidate Class II objects, cyan crosses candidate transition disk objects. 


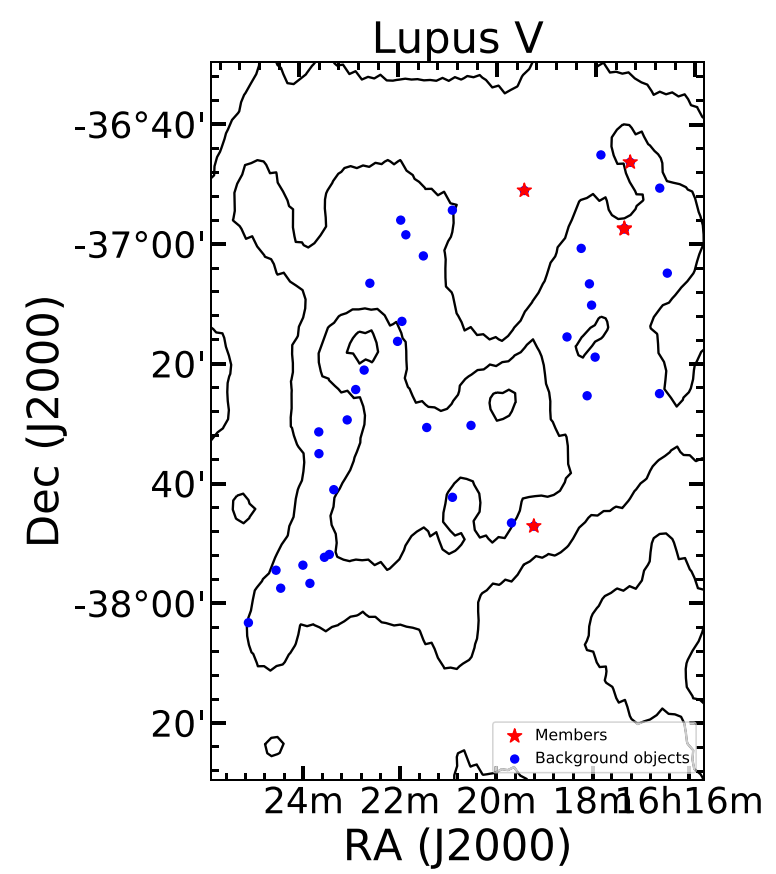

Fig. B.3. Extinction map from IRAS and COBE/DIRBE (Schlegel et al. 1998). Contours correspond to these values of $E(B-V)$ : $0.66,0.91,1.17$, $1.42,1.68$. The symbols of two of the members overlap. 